\title{
A Systematic Review and Meta-Analysis of Endocrine-Related Adverse Events Associated with Immune Checkpoint Inhibitors
}

Authors

Jeroen de Filette ${ }^{1}$, Corina Emilia Andreescu1, Filip Cools² ${ }^{2}$ Bert Bravenboer ${ }^{1}$, Brigitte Velkeniers ${ }^{1}$

Affiliations

1 Department of Endocrinology, Universitair Ziekenhuis Brussel, Vrije Universiteit Brussel, Brussels, Belgium

2 Department of Neonatology, Universitair Ziekenhuis Brussel, Vrije Universiteit Brussel, Brussels, Belgium

\author{
Key words \\ hypophysitis, thyroiditis, CTLA-4, PD-1, PD-L1 \\ received $\quad 11.06 .2018$ \\ accepted 22.01.2019 \\ Bibliography \\ DOI https://doi.org/10.1055/a-0843-3366 \\ Horm Metab Res 2019; 51: 145-156 \\ (c) Georg Thieme Verlag KG Stuttgart · New York \\ ISSN 0018-5043 \\ Correspondence \\ Jeroen de Filette \\ Department of Endocrinology \\ Universitair Ziekenhuis Brussel \\ Vrije Universiteit Brussel \\ Laarbeeklaan 101 \\ 1090 Brussels \\ Belgium \\ Tel.: + 32247760 01, Fax: + 3224776800 \\ jdefilet@vub.ac.be
}

Supplementary Material for this article is available online at http://www.thieme-connect.de/products.

\section{ABSTRACT}

Monoclonal antibodies targeting cytotoxic T-lymphocyte antigen-4 (CTLA-4), programed cell death 1 (PD-1), or its ligand (PD-L1) have become the mainstay for advanced malignancies. The incidence of endocrine adverse events provoked by these immune checkpoint inhibitors (ICI) is based on data from randomized controlled trials, which have their drawbacks. PubMed was searched through August 22nd, 2017, by 2 reviewers independently (J.d.F. and C.E.A.). Early phase I/II, phase III experimental trials, prospective and retrospective observational studies were included. The weighted incidence and risk ratio were estimated for hypophysitis, primary thyroid disease, primary adrenal insufficiency, and diabetes mellitus. Their management is discussed in a systematic review. A total of 101 studies involving 19922 patients were included. Ipilimumab-treated patients experienced hypophysitis in $5.6 \%(95 \% \mathrm{Cl}$, 3.9-8.1), which was higher than nivolumab $(0.5 \% ; 95 \% \mathrm{Cl}$, $0.2-1.2)$ and pembrolizumab (1.1\%; $95 \% \mathrm{Cl}, 0.5-2.6)$. PD-1/ PD-L1 inhibitors had a higher incidence of thyroid dysfunction - particularly hypothyroidism (nivolumab, $8.0 \%$; $95 \% \mathrm{Cl}, 6.4-$ 9.8; pembrolizumab, 8.5\%; 95\% Cl, 7.5-9.7; PD-L1, 5.5\%; $95 \%$ Cl, 4.4-6.8; ipilimumab, 3.8\%; $95 \%$ Cl, 2.6-5.5). Combination therapy was associated with a high incidence of hypothyroidism (10.2-16.4\%), hyperthyroidism (9.4-10.4\%), hypophysitis (8.8-10.5\%), and primary adrenal insufficiency (5.2-7.6\%). Diabetes mellitus and primary adrenal insufficiency were less frequent findings on monotherapy. Our meta-analysis shows a high incidence of endocrine adverse events provoked by single agent checkpoint blockade, further reinforced by combined treatment.

\section{Introduction}

Immune checkpoint inhibitors (ICI) are revolutionizing the management of advanced malignancies. The importance of this new generation of cancer therapy through immune modulation will only expand in the near future, given their recent extended approval [1]. These novel antibodies release the brakes of the immune system and potentiate antitumor immune responses through the inhibition of receptors on immune and cancer cells such as cytotoxic T-lymphocyte antigen 4 (CTLA-4) or programmed cell death 1 (PD1 ) and its ligand (PD-L1), whose function is to maintain self-toler- ance [2]. Their side effects are equally fascinating as immune-related adverse events (irAE) have been described in almost all organs including the endocrine system. The anti-CTLA-4 agent ipilimumab is associated with the otherwise rare phenomenon of hypophysitis, while PD-1 inhibitors nivolumab and pembrolizumab often provoke primary thyroid dysfunction [3]. Notwithstanding, cases of primary adrenal insufficiency, fulminant diabetes mellitus, and hypoparathyroidism associated with checkpoint blockade have also been reported. The aim of the present study is to perform a systematic review and meta-analysis of immune checkpoint inhibitors 
and endocrine side effects, including their incidence, evaluation, and management.

\section{Materials and Methods}

\section{Literature search}

The PubMed database was searched through August 22nd, 2017 , for relevant articles on the subject of endocrinopathies and ICI, by 2 reviewers independently (J.d.F. and C.E.A.). Search terms were included for the various endocrinopathies, adverse events and the currently available ICl: 'ipilimumab', 'tremelimumab', 'nivolumab', 'pembrolizumab', 'atezolizumab', 'avelumab' and 'durvalumab'. These were combined with the Boolean logical operators AND/OR. The authors also checked recently published literature [3-7] and identified 2 additional studies.( $\triangleright$ Table 1,2 )

\section{Study selection}

Early phase I/II, phase III experimental trials, and prospective as well as retrospective observational studies were included. Study subjects had to be adults with any type of advanced, metastatic, or unresectable malignancy. Studies with regimens combining $\mathrm{ICI}$ with radiotherapy, cell vaccines, small molecule inhibitors or immunotherapy with IL-2 or interferon were omitted. Language was restricted to English or French. The investigators used the title and abstract for manuscript selection. Articles were categorized as follows: Clinical Study, Case Report, Review (of endocrine adverse events), Systematic Review or Meta-Analysis, Mechanism (of checkpoint blockade toxicity), Basic or Translational Research, Combination Therapy, Economic or Health-Care, Language, Pediatric, Off Topic. After initial selection, full texts were reviewed and further excluded if no endocrine adverse events were reported or if the safety data were inadequate. Duplicates or studies on the same population were identified through their trial registry number and excluded as well. Any discrepancies were discussed by all authors and resolved by consensus. Supplemental ( $\vee$ Table $1 \mathrm{~S}$ ) provides an overview of all included studies [8-108].

\section{Data analysis and extraction}

The following elements were extracted from each included study: author and year of publication, study design, median follow-up time, treatment and placebo/chemotherapy arms, dosing and frequency of drug administration, endocrine adverse events (hypothyroidism, hyperthyroidism, thyroiditis, hypophysitis, primary adrenal insufficiency, and diabetes mellitus), trial registration number, and trial name. When available, supplementary data and appendices were also methodically explored.

\section{Statistical analysis}

For each study, the incidence of a specific endocrine adverse event was calculated by dividing the number of observed events by the total number of patients that were followed-up during the study period. A meta-analysis of incidence estimates was performed using an inverse variance method to weigh the studies. Summary estimates of incidence are reported with their $95 \%$ confidence interval $(\mathrm{Cl})$. For randomized controlled trials, a relative risk and $95 \%$ $\mathrm{Cl}$ were calculated using the number of observed adverse events and the total number of patients in each group. If possible, relative risks from individual trials for the same adverse event were combined in a meta-analysis. Heterogeneity was assessed using the $\mathrm{Q}$ and $\mathrm{I}^{2}$ statistics. The random-effects model was a priori selected based on the expected study heterogeneity. All analyses were performed with Comprehensive Meta-Analysis v3.0 (Biostat, Inc.).

\section{Results}

\section{Study characteristics}

Our search identified a total of 1169 articles, of which 959 were excluded based on title and abstract. Of the 210 full texts that were reviewed, 101 were finally included ( $\triangleright$ Fig. 1 ). These included 7 phase II, 1 phase II/III, 17 phase III randomized trials, 64 prospective, and 12 retrospective studies. Within the 101 included articles, a total of 152 study arms were identified. The main tumor types were melanoma (69/152, $45 \%)$, non-small cell lung carcinoma (31/152, $20 \%)$, and renal cell carcinoma (11/152, $7 \%)$. A total of 19922 patients were analyzed (ipilimumab, $n=4430$; tremelimumab, $n=1171$; nivolumab, $n=3317$; pembrolizumab, $n=4485$; atezolizumab, $n=998$; avelumab, $n=316$; durvalumab, $n=191$; combination, $n=1078$; placebo/chemotherapy, $n=3936)$. The regimens were classified as monotherapy with anti-CTLA-4 (41/152, $27 \%)$, anti-PD-1 (68/152, 45\%), anti-PD-L1 (8/152, $5 \%$ ), combination of anti-CTLA-4 with anti-PD-1 (13/152, $9 \%)$ or anti-PD-L1 (3/152, 2\%), and placebo/chemotherapy (19/152, 13\%).

\section{Incidence of hypophysitis}

The CTLA-4 inhibitor ipilimumab had a $5.6 \%(95 \% \mathrm{Cl}, 3.9-8.1)$ pooled estimated incidence of hypophysitis vs. $1.8 \%$ ( $95 \% \mathrm{Cl}, 1.1-$ 2.9) for tremelimumab. Nivolumab and pembrolizumab had a lower incidence at $0.5 \%(95 \% \mathrm{Cl}, 0.2-1.2)$ and $1.1 \%(95 \% \mathrm{Cl}, 0.5-$ 2.6), respectively. The highest estimated incidence was noted on combination therapy, ranging from $8.8 \%(95 \% \mathrm{Cl}, 6.2-12.4)$ to $10.5 \%$ (95\% Cl, 6.5-16.4). No data on hypophysitis were available for anti-PD-L1 agents. Compared to patients not receiving immunotherapy, ipilimumab-treated patients had a higher risk of developing hypophysitis ( $R R, 9.26 ; 95 \% \mathrm{Cl}, 0.51-169.66 ; \mathrm{p}=0.134)$. This was lower for nivolumab (RR, 2.06; $95 \% \mathrm{Cl}, 0.22-19.68 ; \mathrm{p}=0.531$ ) and for pembrolizumab (RR, 2.89; $95 \% \mathrm{Cl}, 0.46-18.27$; $\mathrm{p}=0.259$ ). These results were, however, not statistically different.

\section{Incidence of primary thyroid disease}

Checkpoint blockade with PD-1 or PD-L1 inhibitors was particularly associated with primary thyroid dysfunction. Hypothyroidism was most frequent, followed by hyperthyroidism and thyroiditis. The summary estimated incidence of hypothyroidism was $8.0 \%$ (95\% Cl, 6.4-9.8) for nivolumab, $8.5 \%$ (95\% Cl, 7.5-9.7) for pembrolizumab, $6.0 \%(95 \% \mathrm{Cl}, 4.2-8.4)$ for atezolizumab, 5.5\% (95\% $\mathrm{Cl}, 3.5-8.7)$ for avelumab, and $4.7 \%(95 \% \mathrm{Cl}, 2.5-8.8)$ for durvalumab. Ipilimumab had a lower estimated incidence of $3.8 \%$ (95\% $\mathrm{Cl}, 2.6-5.5)$ while clear data on tremelimumab were missing. Regardless, one study reported up to $5.2 \%$ of thyroid disorders on tremelimumab [41]. Combination therapy had the highest estimated incidence of hypothyroidism with $10.2 \%$ (95\% Cl, 5.6-17.9) for durvalumab with tremelimumab, $15.1 \%(95 \% \mathrm{Cl}, 10.6-21.8)$ for 


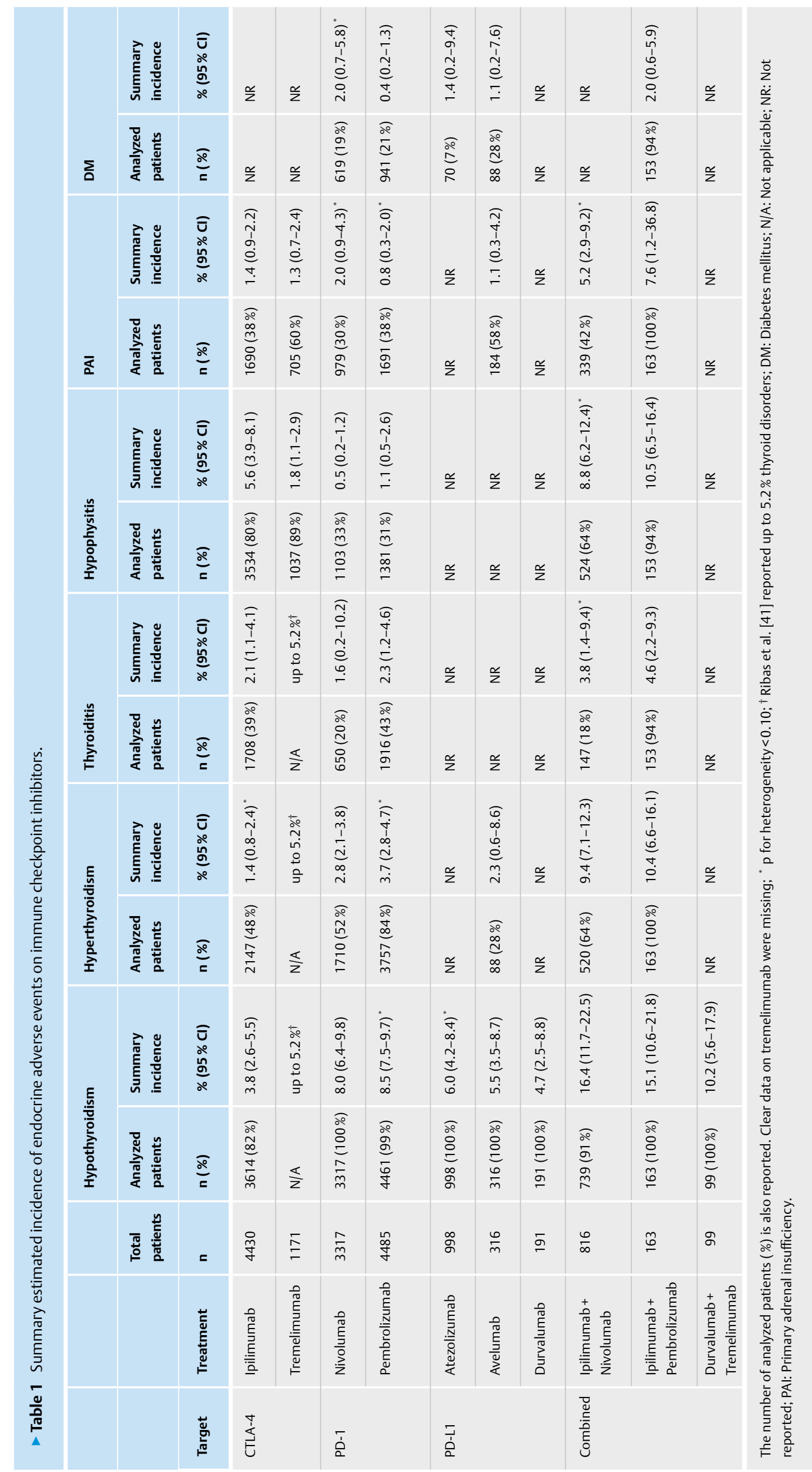


- Table 2 Summary of relative risk for endocrine adverse events.

\begin{tabular}{|c|c|c|c|c|c|c|c|c|}
\hline \multirow[b]{2}{*}{ Treatment } & \multicolumn{2}{|l|}{ Hypothyroidism } & \multicolumn{2}{|l|}{ Hyperthyroidism } & \multicolumn{2}{|l|}{ Thyroiditis } & \multicolumn{2}{|l|}{ Hypophysitis } \\
\hline & $\%(95 \% \mathrm{Cl})$ & p-value & $\%(95 \% \mathrm{Cl})$ & p-Value & $\%(95 \% \mathrm{Cl})$ & p-Value & $\%(95 \% \mathrm{Cl})$ & p-Value \\
\hline $\begin{array}{l}\text { Ipilimumab vs. } \\
\text { placebo/chemo }\end{array}$ & $6.51(3.37-12.58)$ & $<0.001$ & $6.83(0.81-57.83)$ & 0.078 & $5.08(0.25-105.29)$ & 0.293 & $9.26(0.51-169.66)$ & 0.134 \\
\hline $\begin{array}{l}\text { Nivolumab vs. } \\
\text { placebo/chemo }\end{array}$ & $11.19(4.31-29.08)$ & $<0.001$ & $4.20(1.12-15.84)$ & 0.034 & $2.56(0.28-23.08)$ & 0.402 & $2.06(0.22-19.68)$ & 0.531 \\
\hline $\begin{array}{l}\text { Pembrolizumab vs. } \\
\text { placebo/chemo }\end{array}$ & $7.41(4.06-13.52)$ & $<0.001$ & $8.52(3.66-19.84)$ & $<0.001$ & $6.56(0.80-53.61)$ & 0.079 & $2.89(0.46-18.27)$ & 0.259 \\
\hline
\end{tabular}

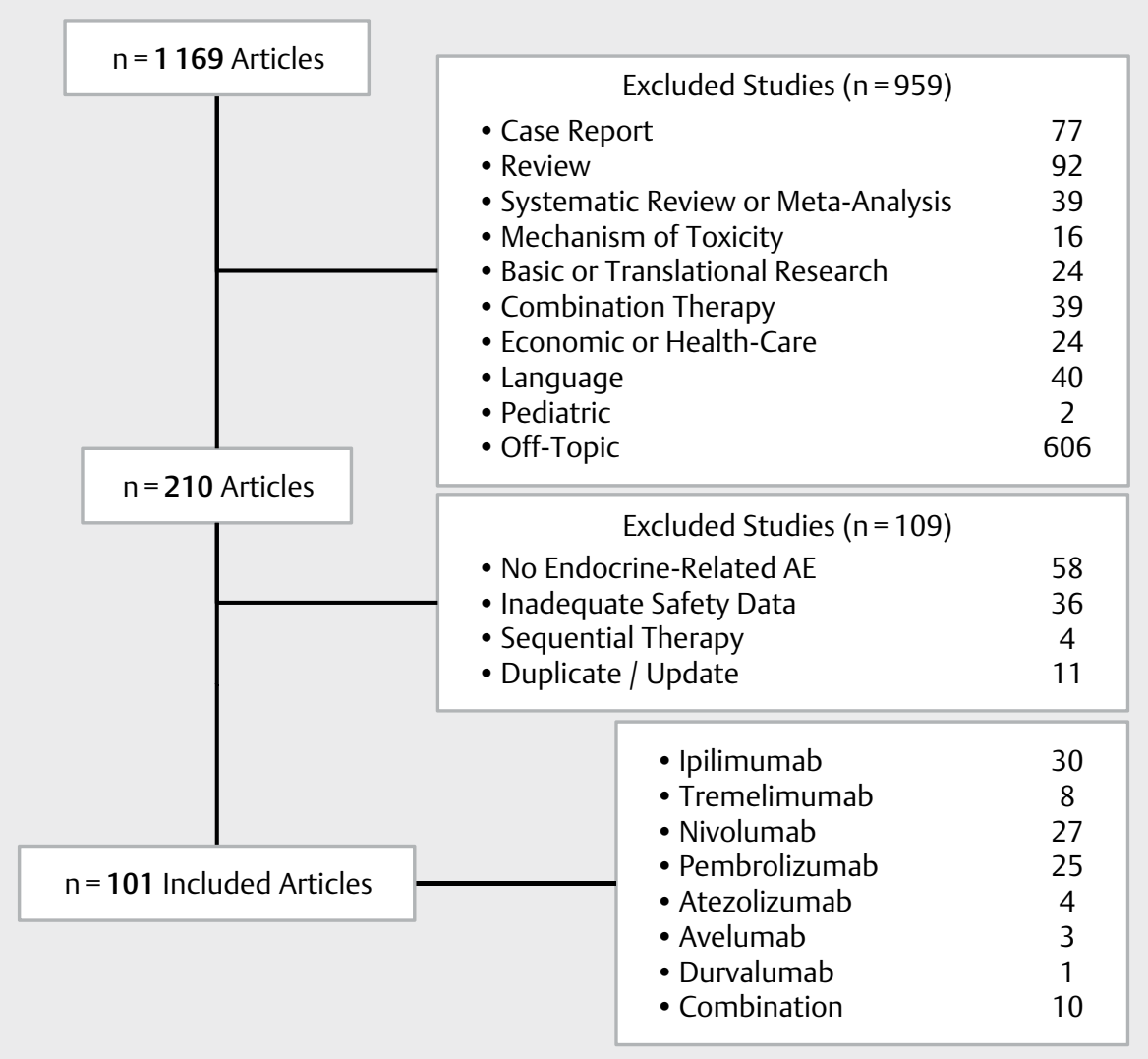

Fig. 1 Flow chart of study selection.

ipilimumab with pembrolizumab and $16.4 \%$ (95\% CI, 11.7-22.5) for ipilimumab with nivolumab. Compared to placebo/chemotherapy, the risk of hypothyroidism was significantly increased by all immunotherapies. The PD-1 inhibitor nivolumab (RR, 11.19; $95 \%$ $\mathrm{Cl}, 4.31-29.08 ; \mathrm{p}<0.001)$ had a higher risk than pembrolizumab (RR, 7.41; $95 \% \mathrm{Cl}, 4.06-13.52 ; \mathrm{p}<0.001$ ) or ipilimumab (RR, 6.51; $95 \%$ Cl, 3.37-12.58; $\mathrm{p}<0.001)$.

Predictions for hyperthyroidism were lower with a pooled incidence of $2.8 \%$ ( $95 \% \mathrm{Cl}, 2.1-3.8)$ for nivolumab, 3.7\% (95\% Cl, 2.84.7) for pembrolizumab, $2.3 \%(95 \% \mathrm{Cl}, 0.6-8.6)$ for avelumab, and $1.4 \%(95 \% \mathrm{Cl}, 0.8-2.4)$ for ipilimumab. No data were available for atezolizumab and durvalumab. For combination therapy, the estimated incidence was $9.4 \%(95 \% \mathrm{Cl}, 7.1-12.3)$ for ipilimumab with nivolumab and $10.4 \%(95 \% \mathrm{Cl}, 6.6-16.1)$ for ipilimumab with pembrolizumab. As compared to placebo/chemotherapy, the risk of developing hyperthyroidism increased with nivolumab (RR, 4.20; $95 \%$ $\mathrm{Cl}, 1.12-15.84 ; \mathrm{p}=0.034)$, pembrolizumab (RR, 8.52; $95 \% \mathrm{Cl}$, 3.66-19.84, $\mathrm{p}<0.001$ ), and ipilimumab (RR, 6.83; $95 \% \mathrm{Cl}, 0.81-$ 57.83; $p=0.078$ ), although the latter was not significant.

Few data on thyroiditis were available. Estimations ranged from $1.6 \%(95 \% \mathrm{Cl}, 0.2-0.2)$ for nivolumab, $2.3 \%$ (95\% Cl, 1.2-4.6) for pembrolizumab, $2.1 \%(95 \% \mathrm{Cl}, 1.1-4.1)$ for ipilimumab, $3.8 \%(95 \%$ 
Cl, 1.4-9.4) for ipilimumab with nivolumab to $4.6 \%$ (95\% Cl, 2.29.3) for ipilimumab with pembrolizumab. Nivolumab (RR, 2.56; $95 \%$ $\mathrm{Cl}, 0.28-23.08$; $\mathrm{p}=0.402)$, pembrolizumab (RR, 6.56; $95 \% \mathrm{Cl}, 0.80-$ 53.61; $\mathrm{p}=0.079$ ), and ipilimumab (RR, 5.08; $95 \% \mathrm{Cl}, 0.25-105.29$; $\mathrm{p}=0.293$ ) presented a higher risk, although not significantly.

\section{Incidence of diabetes mellitus and primary adrenal insufficiency}

Primary adrenal insufficiency had a predicted incidence of $1.4 \%$ (95\% Cl, 0.9-2.2) on ipilimumab, $1.3 \%(95 \% \mathrm{Cl}, 0.7-2.4)$ on tremelimumab, $2.0 \%(95 \% \mathrm{Cl}, 0.9-4.3)$ on nivolumab, and $0.8 \%$ ( $95 \% \mathrm{Cl}, 0.3-2.0)$ on pembrolizumab monotherapy. For combination therapy, estimations ranged from $5.2 \%(95 \% \mathrm{Cl}, 2.9-9.2)$ to $7.6 \%$ ( $95 \% \mathrm{Cl}, 1.2-36.8$ ) for ipilimumab with nivolumab, or pembrolizumab, respectively.

Diabetes mellitus was mainly PD-1/PD-L1 related without cases observed on CTLA-4 therapy. The incidence was $2.0 \%(95 \% \mathrm{Cl}, 0.7-$ $5.8)$ for nivolumab and $0.4 \%(95 \% \mathrm{Cl}, 0.2-1.3)$ for pembrolizumab. No further analyses were performed due to the rarity of these side effects.

\section{Discussion}

Our meta-analysis shows a high incidence of all-grade endocrine adverse events related to immune checkpoint therapy, which is further enhanced by combined treatment. The highest incidence of hypophysitis on monotherapy is noted on anti-CTLA-4 therapy with ipilimumab (but not with tremelimumab) although hypophysitis also develops during PD-1 blockade. The incidence of hypothyroidism on monotherapy is highest for PD- 1 inhibitors, followed by PD-L1 and CTLA-4 blockade. Hyperthyroidism and thyroiditis occur less frequently. Primary adrenal insufficiency and diabetes mellitus are less frequent, with no cases of diabetes mellitus reported on anti-CTLA-4 therapy. Combined ICI shows a remarkably higher incidence of hypothyroidism, hyperthyroidism, hypophysitis, and primary adrenal insufficiency.

\section{Hypophysitis}

Our analysis indicates an higher incidence of all-grade hypophysitis in patients treated with ipilimumab. Anti-CTLA-4-induced hypophysitis usually occurs after 4 to 10 weeks of treatment (usually after the third infusion). In one report, hypophysitis was more frequent among patients receiving a higher dose of ipilimumab $(10 \mathrm{mg} / \mathrm{kg})$ with a 2 -fold risk increase compared to patients treated with lower dose $(3 \mathrm{mg} / \mathrm{kg}$ ) [8]. Hypophysitis was more frequent among older and male patients [18, 25]. Anti-PD-1 or PD-L1 treatment rarely induced hypophysitis or hypopituitarism. These findings extend the data of previous reviews [6, 8, 109-111]. Hypophysitis may be life threatening due to the abrupt onset of central adrenal failure. The initial presentation is indolent and includes symptoms such as headache and fatigue. Hormonal and radiological evaluation of the pituitary should be performed. The pituitary is often diffusely enlarged in the acute phase, with or without stalk thickening. Homogeneous or heterogeneous pituitary gland enhancement is possible. This is followed by a gradual decrease in size of the pituitary with partial or complete loss of pituitary function $[25,27,112]$. Hence, normal imaging of the pituitary does not ex- clude its diagnosis [27]. Corticotropic, thyrotropic and gonadotropic functions are mostly affected, but long term failure of the corticotropic axis is paramount $[10,25,112]$. High-dose glucocorticoids are proposed for those with mass effect symptoms, such as severe headache or visual-field disturbance $[27,113,114]$. Their role in the prevention of secondary adrenal failure has, however, been challenged and it is possible that physiological corticosteroid substitution suffices $[18,25,112]$. The potential benefit of highdose glucocorticoid treatment should be balanced against the loss of efficacy resulting from the anti-cancer immunotherapy, although this issue is controversial. Why ACTH, TSH, and gonadotropic cells are particularly vulnerable to destruction with anti-CTLA- $4 \mathrm{mAb}$ is not fully elucidated. The expression of CTLA-4 on ACTH- and TSH-secreting cells has been implicated through immunohistochemistry $[115,116]$. Radiological mimicry with lymphocytic hypophysitis favors the hypothesis of a lymphocytic destruction of the pituitary [27] as a result of T cell-mediated cytotoxicity, but an anti-pituitary antibody mediated process cannot be excluded. Iwama et al. found that all 7 patients with hypophysitis developed antibodies recognizing predominantly TSH- (7/7), FSH- (5/7), and ACTH-secreting (3/7) cells [115]. The diagnostic accuracy of these pituitary antibodies is not yet known. An autopsy study suggests that administration of CTLA-4 blocking antibodies to patients who express high levels of pituitary CTLA-4 antigen causes a necrotizing form of hypophysitis through type II (IgG dependent) and type IV (T-cell dependent) immune mechanisms [116]. The difference in incidence between ipilimumab ( $\lg G 1)$ and tremelimumab ( $\lg G 2)$ is probably due to their different immunological subclasses. In humans, polymorphisms in the CTLA4 gene confer increased susceptibility to a variety of autoimmune diseases, including Hashimoto's thyroiditis, type 1 diabetes mellitus, and Addison's disease $[117,118]$. This polymorphism has not been analyzed in patients developing hypophysitis on CTLA-4 blockade. Some data associate the endocrine and other immune-related adverse events to a better outcome of anti-cancer immunotherapy [25]. In one study, ipilimumab $10 \mathrm{mg} / \mathrm{kg}$ resulted in significantly longer overall survival against ipilimumab $3 \mathrm{mg} / \mathrm{kg}$ but with increased treatment-related adverse events including hypophysitis [111]. In summary, patients who receive immune checkpoint inhibitors, particularly anti-CTLA-4 therapy, should be carefully evaluated to detect pituitary-related side effects. In case of hypophysitis, adequate hormone replacement therapy should be initiated. Failure of the corticotropic axis is usually permanent and requires continuous glucocorticoid substitution therapy, while failure of thyrotropic and gonadotropic axes may be transient.

\section{Thyroid dysfunction}

Checkpoint blockade with PD-1 or PD-L1 inhibitors is particularly associated with primary thyroid dysfunction. The spectrum of thyroid side effects includes primary hypo-, hyperthyroidism, and painless thyroiditis. The authors define painless thyroiditis as thyrotoxicosis followed by euthyroidism or hypothyroidism, with negative TRAb, reduced or absent tracer uptake on technetium scan and/or increased 18fluorodeoxyglucose uptake on positron emission tomography ( ${ }^{18}$ FDG-PET). The authors define hyperthyroidism as a suppressed TSH with an elevated fT4 and/or fT3 level, and hypothyroidism as an elevated TSH with a decreased fT4 level. Importantly, a preceding phase 
of hyperthyroidism cannot be excluded in case of hypothyroidism, unless thyroid function tests were systematically followed. Painless thyroiditis can occur early-on, within the first weeks of checkpoint blockade therapy $[83,119,120]$. The initial phase of thyrotoxicosis (suppressed TSH and high fT4) evolves within 3-6 weeks to hypothyroidism (high TSH and low fT4) $[75,83,119,120]$. Symptoms of thyrotoxicosis are rarely severe. The underlying process is likely to be a destructive, inflammatory thyroiditis. Thyroglobulin ( $\mathrm{Tg}$ ) levels, while not specific, were elevated during thyrotoxicosis and normalized with subsequent hypothyroidism in 5 patients with nivolumab-induced thyroiditis [120]. Diffuse increased uptake of the inflammatory tracer ${ }^{18} \mathrm{FDG}$ in the thyroid gland was observed in pembrolizumab-induced thyroiditis [ 75,83$]$ and in 2 transiently thyrotoxic patients on nivolumab [121]. The differential diagnosis of thyroid dysfunction on checkpoint blockade might be difficult. Hypothyroidism secondary to pituitary failure should be excluded as thyroid substitution therapy could precipitate adrenal failure. A low to normal TSH with low fT4 should prompt the suspicion of hypopituitarism. Euthyroid sick syndrome is also possible in the setting of advanced malignancy. Hyperthyroidism usually results from destructive thyroiditis but cases of Graves' disease on ipilimumab and tremelimumab have been described [122-124]. Iodine contamination due to frequent radiological investigations and subsequent iodine-induced hyperthyroidism is another possibility in this patient population. Measurement of TSH-receptor stimulating antibodies, thyroid scintigraphy and Doppler flow ultrasonography have their use in distinguishing thyroiditis (low uptake on scintigraphy and low blood flow on ultrasound) from Graves' disease (high uptake on scan and high blood flow on ultrasound). Of course, recent administration of iodine-containing contrast media could suppress the uptake measured in thyroid scintigraphy [125]. Symptomatic treatment with non-selective beta-blockers could be required. The duration of hyperthyroidism due to thyroiditis is usually short and no additional therapy is required. Antithyroid drugs (e.g., methimazole, propylthiouracil) should be started on suspicion of Graves' disease or in high-grade hyperthyroidism while awaiting further investigations. Glucocorticosteroids have been used in a patient with thyroid storm [126]. As in hypophysitis, their use is controversial and should only be considered in the most severe cases. Thyroid hormone substitution is usually required for the subsequent phase of hypothyroidism, although a select number of cases were reversible [75, 127].

Why anti-PD-1/PD-L1 therapy favors thyroid dysfunction is not known. The expression of PD-L1 and PD-L2 in normal thyroid tissue has only been analyzed recently [128] and suggests a distinct immunomodulatory role in the thyroid gland. Increased PD-L1 expression has been identified in papillary [129] and anaplastic thyroid carcinoma [130] as a plausible mechanism to evade antitumor immune responses. Polymorphisms in the genes encoding PD-1 and its ligand are related to Graves' disease, but their association is weak $[131,132]$. It remains to be defined whether the subpopulation with preexisting subclinical autoimmune thyroid disease is at any particular risk. The absence of pretreatment thyroid function and antithyroid antibody levels is a limitation in establishing the baseline susceptibility of these patients. Thyroperoxidase antibodies (TPOAb), identified after anti-PD-1 treatment in patients with thyroid dysfunction, have also been identified at baseline in some studies $[75,83,119,120]$. These findings have only recently been verified in a prospective study, wherein the presence of thy- roid autoantibodies seems to increase the risk for thyroid dysfunction [133].

As a side note, treatment with L-T3 may be a better choice than L-T4. The presence of hypothyroidism could have beneficial effects on tumor behavior. As pointed out in a review by Moeller et al. [134], observations in mouse models suggest that hypothyroidism induced by propylthiouracil suppresses tumor growth, whereas T4 administration had the opposite effect $[135,136]$. In a murine model of lung carcinoma, treatment with T3 suppressed metastatic tumor growth and prolonged survival while T4 enhanced tumor growth [137]. In humans, hypothyroidism induced by treatment with IL-2 or the tyrosine-kinase inhibitor sunitinib was associated with a prolonged progression-free survival $[138,139]$ or response to treatment [140]. In an observational study of 23 patients, euthyroid hypothyroxinemia was achieved by the administration of methimazole with the addition of L-T3, while preexisting hypothyroid patients were treated with T3 for T4 replacement. Most of these patients (19 of 23) had a prolonged survival when compared to epidemiological data [141]. It is currently unknown if and how these findings should be implemented in routine clinical practice. There is an urgent need for prospective evaluation of tumor burden with L-T3 compared to L-T4 replacement and to define the optimal level of thyroid hormone substitution balancing the quality of life and the stimulatory effects on tumor growth.

\section{Diabetes mellitus}

While hypophysitis and thyroid disorders are predominant, checkpoint-blockade associated diabetes mellitus deserves further notice. Its presentation is often severe with fulminant diabetes and ketoacidosis [142]. Its onset ranges from a few weeks [143] up to one year after initiating therapy [144]. The clinical presentation consists of classic symptoms with polyuria, polydipsia, fatigue, weight loss, and dehydration. The C-peptide level is low or undetectable while HbA1c levels are not always very elevated (e. g., $<53$ $\mathrm{mmol} / \mathrm{mol}$ ). Diagnostic antibodies found in type 1 diabetes are detected in only half of cases $[142,145]$. This could be due to the acute onset of beta cell inflammation. Dysregulation of PD-1 is implied in both checkpoint-blockade and conventional type 1 diabetes, as peripheral $\mathrm{CD}^{+}{ }^{+}$- and regulatory $T$ cells of type 1 diabetes patients show a reduced PD- 1 expression $[146,147]$. Interestingly, a low CTLA-4 expression on CD4 ${ }^{+}$- and regulatory $T$ cells is also found in type 1 diabetes $[148,149]$, suggesting that patients receiving CTLA-4 blocking mAb are also susceptible. Hyperreactivity of the immune system is suspected as some patients experience thyroiditis before the onset of diabetes $[143,145]$. This could be in part explained by HLA haplotypes associated with type 1 diabetes and perhaps also with checkpoint-blockade induced diabetes mellitus. The reverse could also be true as one Japanese patient, carrier of an HLA allele resistant to type 1 diabetes, was less severely affected, without ketoacidosis nor complete insulin deficiency [142]. Long-term treatment consists of insulin therapy. The beta-cell destruction induced by checkpoint inhibitors is unlikely to be reversible. However, longer follow-up is required. Screening measures should include routine measurement of blood glucose and HbA1c. Chronic complications should be monitored in those with a survival lasting more than 5 years. 


\section{Primary adrenal insufficiency}

Checkpoint-blockade associated adrenal insufficiency is a potentially life-threatening complication, caused by hypophysitis or adrenalitis in this patient population. Symptoms are non-specific and include nausea, fatigue, anorexia, abdominal pain, weight loss, hypotension and hypoglycemia. Low early morning serum cortisol is abnormal and the concomitant presence of a high serum ACTH is suggestive of primary adrenal insufficiency, while low serum ACTH is suggestive of secondary adrenal insufficiency. These findings should prompt dynamic testing. The identification of primary adrenal insufficiency can be difficult however by the concomitant use of corticosteroids in the treatment of other irAE. The incidence of combined primary and secondary adrenal insufficiency is also unknown. Primary adrenal insufficiency is probably mediated by adrenalitis. Adrenal autoantibodies were detected in one case of pembrolizumab-induced adrenal failure [150] while bilateral adrenal gland enlargement [151] and increased bilateral ${ }^{18}$ FDG activity [152] have also been observed. Routine screening of pituitary function tests is advisable before each dosing of immune checkpoint therapy. Patients should be informed about the potential danger of cortisol deficiency. In case of primary adrenal insufficiency, patients should not only receive gluco- but also mineralocorticosteroids.

\section{Other endocrine events}

Few data are available on the gonadal and parathyroid function during checkpoint blockade therapy. In one retrospective review, 9 ipilimumab-treated patients were identified with low testosterone levels in the absence of hypophysitis [27]. Hypogonadism is difficult to interpret in this patient population because of the severe illness in malignancy and associated secondary hypogonadotropic hypogonadism as well as the use of exogenous corticosteroids for the treatment of irAE. Human fertility could potentially be impaired in the event of hypophysitis leading to persistently low FSH and LH levels. Incidental hypercalcemia with low PTH levels was found in 2 patients [27], perhaps due to hypercalcemia of malignancy. Primary hypoparathyroidism with acute hypocalcemia was described in one case [153], while hypoparathyroidism with no information on serum calcium was detected in one other patient [154].

\section{Study design and limitations}

This is a comprehensive analysis of endocrine adverse events on immune checkpoint inhibitors, and the first to include retrospective, prospective, and randomized clinical trials. We chose this approach because adverse events are generally underestimated in randomized clinical trials [155]. The term 'thyroiditis' was not routinely used. Patients with painless thyroiditis can be detected both in the phase of hyperthyroidism or hypothyroidism, further complicating correct reporting. Furthermore, the interpretation of thyroid dysfunction depends of the definition used in each individual study. While the Common Terminology Criteria for Adverse Events (CTCAE) are in widespread use [156], they are imprecise for endocrine disorders, as these do not include a description of the hormone levels nor the separate entity of painless thyroiditis. We are aware that our study has its limitations. The analysis was performed at the study level and did not include individual patient data. Human error cannot be excluded in case of missing studies or duplicates. The analyses of the relative risks were not significant for thyroiditis and hypophysitis, and were generally associated with large confidence intervals. This could be due to the smaller number of patients as prospective and retrospective studies were not included for these investigations. Finally, trials were excluded if the endocrine adverse events were not reported and this may overestimate our final results.

\section{Conclusions}

Hypophysitis is a frequent endocrine adverse event triggered by anti-CTLA-4 mAb, while thyroid dysfunction is commonly observed with anti-PD-1/PD-L1 therapy. Combined checkpoint blockade shows a remarkably higher incidence. Symptoms are often non-specific and may be related to the disseminated cancer or the use of checkpoint inhibitors itself. A high suspicion of endocrine adverse events is therefore warranted as timely diagnosed and treated, one can avoid life-threatening complications. Our knowledge regarding the pathophysiology in the setting of immune checkpoint inhibitors remains limited, but new data start to unravel the mechanisms of immune dysregulation. This could not only be of value to better understand these adverse events, but also the autoimmune endocrine disorders in general.

\section{Funding}

This research did not receive any grant from any funding agency in the public, commercial, or non-profit sector.

\section{Conflict of Interest}

The authors declare that they have no conflict of interest.

\section{References}

[1] U.S. Food and Drug Administration. Hematology/Oncology (Cancer) Approvals \& Safety Notifications.May 262017 Available at https:// www.fda.gov/drugs/informationondrugs/approveddrugs/ ucm279174.htm Accessed June 5, 2017

[2] Abbas AK, Lichtman AH, Pillai S. Cellular and Molecular Immunology. 8th Edition Philadelphia, PA: Elsevier Saunders; 2015

[3] Byun D], Wolchok JD, Rosenberg LM et al. Cancer immunotherapy immune checkpoint blockade and associated endocrinopathies. Nat Rev Endocrinol 2017; 13: 195-207

[4] Barroso-Sousa R, Barry WT, Garrido-Castro AC et al. Incidence of endocrine dysfunction following the use of different immune checkpoint inhibitor regimens: A systematic review and meta-analysis. JAMA Oncol 2018; 4: 173-182

[5] Wang PF, Chen Y, Song SY et al. Immune-related adverse events associated with anti-PD-1/PD-11 treatment for malignancies: A meta-analysis. Front Pharmacol 2017; 8: 730

[6] Shang YH, Zhang $\mathrm{Y}$, Li JH et al. Risk of endocrine adverse events in cancer patients treated with PD-1 inhibitors: A systematic review and meta-analysis. Immunotherapy 2017; 9: 261-272

[7] Abdel-Rahman O, ElHalawani H, Fouad M. Risk of endocrine complications in cancer patients treated with immune check point inhibitors: A meta-analysis. Future Oncol (London, England) 2016; 12: $413-425$ 
[8] Ascierto PA, Del Vecchio M, Robert C et al. Ipilimumab $10 \mathrm{mg} / \mathrm{kg}$ versus ipilimumab $3 \mathrm{mg} / \mathrm{kg}$ in patients with unresectable or metastatic melanoma: A Randomised, double-blind, multicentre, phase 3 trial. Lancet Oncol 2017; 18: 611-622

[9] Bang Y], Cho JY, Kim YH et al. Efficacy of sequential ipilimumab monotherapy versus best supportive care for unresectable locally advanced/metastatic gastric or gastroesophageal junction cancer. Clin Cancer Res 2017; 23: 5671-5678

[10] Brilli L, Danielli R, Ciuoli C et al. Prevalence of hypophysitis in a cohort of patients with metastatic melanoma and prostate cancer treated with ipilimumab. Endocrine 2017; 58: 535-541

[11] Jung M, Lee J, Kim TM et al. Ipilimumab real-world efficacy and safety in korean melanoma patients from the korean named-patient program cohort. Cancer Res Treat 2017; 49: 44-53

[12] Schachter ], Ribas A, Long GV et al. Pembrolizumab versus ipilimumab for advanced melanoma: Final overall survival results of a multicentre, randomised, open-label phase 3 study (KEYNOTE-006). Lancet (London, England) 2017; 390: 1853-1862

[13] Wen X, Ding Y, Li J et al. The experience of immune checkpoint inhibitors in Chinese patients with metastatic melanoma: A retrospective case series. Cancer Immunol Immunother 2017; 66: 1153-1162

[14] Boudewijns S, Koornstra RH, Westdorp H et al. Ipilimumab administered to metastatic melanoma patients who progressed after dendritic cell vaccination. Oncoimmunology 2016; 5: e1201625

[15] Eggermont AM, Chiarion-Sileni V, Grob JJ et al. Prolonged survival in stage III melanoma with ipilimumab adjuvant therapy. N Engl J Med 2016; 375: 1845-1855

[16] Hodi FS, Chesney J, Pavlick AC et al. Combined nivolumab and ipilimumab versus ipilimumab alone in patients with advanced melanoma: 2-year overall survival outcomes in a multicentre, randomised, controlled, phase 2 trial. Lancet Oncol 2016; 17 : 1558-1568

[17] Larkin J, Chiarion-Sileni V, Gonzalez R et al. Combined Nivolumab and Ipilimumab or Monotherapy in Untreated Melanoma. N Engl J Med 2015; 373: 23-34

[18] Min L, Hodi FS, Giobbie-Hurder A et al. Systemic high-dose corticosteroid treatment does not improve the outcome of ipilimumab-related hypophysitis: A retrospective cohort study. Clin Cancer Res 2015; 21: 749-755

[19] Yamazaki N, Kiyohara Y, Uhara H et al. Phase II study of ipilimumab monotherapy in Japanese patients with advanced melanoma. Cancer Chemother Pharmacol 2015; 76: 997-1004

[20] Yamazaki N, Uhara H, Fukushima S et al. Phase II study of the immune-checkpoint inhibitor ipilimumab plus dacarbazine in Japanese patients with previously untreated, unresectable or metastatic melanoma. Cancer Chemother Pharmacol 2015; 76: 969-975

[21] Zimmer L, Eigentler TK, Kiecker $F$ et al. Open-label, multicenter, single-arm phase II DeCOG-study of ipilimumab in pretreated patients with different subtypes of metastatic melanoma. J Transl Med 2015; 13: 351

[22] Zimmer L, Vaubel J, Mohr P et al. Phase II DeCOG-study of ipilimumab in pretreated and treatment-naive patients with metastatic uveal melanoma. PloS One 2015; 10: e0118564

[23] Alexander M, Mellor JD, McArthur $\mathrm{G}$ et al. Ipilimumab in pretreated patients with unresectable or metastatic cutaneous, uveal and mucosal melanoma. Med J Australia 2014; 201: 49-53

[24] Chiarion-Sileni V, Pigozzo J, Ascierto PA et al. Ipilimumab retreatment in patients with pretreated advanced melanoma: The expanded access programme in Italy. Brit Journal Cancer 2014; 110: 1721-1726

[25] Faje AT, Sullivan R, Lawrence D et al. Ipilimumab-induced hypophysitis: A Detailed longitudinal analysis in a large cohort of patients with metastatic melanoma. J Clin Endocrinol Metab 2014; 99: 4078-4085
[26] Kwon ED, Drake CG, Scher HI et al. Ipilimumab versus placebo after radiotherapy in patients with metastatic castration-resistant prostate cancer that had progressed after docetaxel chemotherapy (CA184043): A multicentre, randomised, double-blind, phase 3 trial. Lancet Oncol 2014; 15: 700-712

[27] Ryder M, Callahan M, Postow MA et al. Endocrine-related adverse events following ipilimumab in patients with advanced melanoma: A comprehensive retrospective review from a single institution. Endocr Relat Cancer 2014; 21: 371-381

[28] Altomonte M, Di Giacomo A, Queirolo P et al. Clinical experience with ipilimumab $10 \mathrm{mg} / \mathrm{kg}$ in patients with melanoma treated at Italian centres as part of a European expanded access programme. J Exp Clin Cancer Res 2013; 32: 82

[29] Delyon J, Mateus C, Lefeuvre D et al. Experience in daily practice with ipilimumab for the treatment of patients with metastatic melanoma: An early increase in lymphocyte and eosinophil counts is associated with improved survival. Ann Oncol 2013; 24: 1697-1703

[30] Luke J], Callahan MK, Postow MA et al. Clinical activity of ipilimumab for metastatic uveal melanoma: A retrospective review of the Dana-Farber Cancer Institute, Massachusetts General Hospital, Memorial Sloan-Kettering Cancer Center, and University Hospital of Lausanne experience. Cancer. 2013: 119: 3687-3695

[31] Postow MA, Luke J], Bluth MJ et al. Ipilimumab for patients with advanced mucosal melanoma. Oncologist 2013; 18: 726-732

[32] Robert C, Thomas L, Bondarenko I et al. Ipilimumab plus dacarbazine for previously untreated metastatic melanoma. N Engl ] Med 2011; 364: $2517-2526$

[33] Hodi FS, O'Day SJ, McDermott DF et al. Improved survival with ipilimumab in patients with metastatic melanoma. $\mathrm{N}$ Engl J Med 2010; 363: 711-723

[34] Ku GY, Yuan J, Page DB et al. Single-institution experience with ipilimumab in advanced melanoma patients in the compassionate use setting: Lymphocyte count after 2 doses correlates with survival. Cancer 2010; 116: 1767-1775

[35] Royal RE, Levy C, Turner $\mathrm{K}$ et al. Phase 2 trial of single agent Ipilimumab (anti-CTLA-4) for locally advanced or metastatic pancreatic adenocarcinoma. J Immunother (Hagerstown, Md: 1997) 2010; 33: 828-833

[36] Downey SG, Klapper JA, Smith FO et al. Prognostic factors related to clinical response in patients with metastatic melanoma treated by CTL-associated antigen-4 blockade. Clin Cancer Res 2007; 13 : 6681-6688

[37] Maker AV, Yang JC, Sherry RM et al. Intrapatient dose escalation of anti-CTLA-4 antibody in patients with metastatic melanoma. Journal of immunotherapy (Hagerstown, Md: 1997) 2006; 29: 455-463

[38] Maio M, Scherpereel A, Calabro L et al. Tremelimumab as second-line or third-line treatment in relapsed malignant mesothelioma (DETERMINE): A multicentre, international, randomised, double-blind, placebo-controlled phase 2b trial. Lancet Oncol 2017; 18: 1261-1273

[39] Joshua AM, Monzon JG, Mihalcioiu C et al. A phase 2 study of tremelimumab in patients with advanced uveal melanoma. Melanoma Res 2015; 25: 342-347

[40] Aglietta M, Barone C, Sawyer MB et al. A phase I dose escalation trial of tremelimumab $(C P-675,206)$ in combination with gemcitabine in chemotherapy-naive patients with metastatic pancreatic cancer. Ann Oncol 2014; 25: 1750-1755

[41] Ribas A, Kefford R, Marshall MA et al. Phase III randomized clinical trial comparing tremelimumab with standard-of-care chemotherapy in patients with advanced melanoma. J Clin Oncol 2013; 31: 616-622

[42] Chung KY, Gore I, Fong L et al. Phase II study of the anti-cytotoxic T-lymphocyte-associated antigen 4 monoclonal antibody, tremelimumab, in patients with refractory metastatic colorectal cancer. J Clin Oncol 2010; 28: 3485-3490 
[43] Kirkwood JM, Lorigan P, Hersey P et al. Phase II trial of tremelimumab $(\mathrm{CP}-675,206)$ in patients with advanced refractory or relapsed melanoma. Clin Cancer Res 2010; 16: 1042-1048

[44] Camacho LH, Antonia S, Sosman J et al. Phase I/II trial of tremelimumab in patients with metastatic melanoma. J Clin Oncol 2009; 27: 1075-1081

[45] Ribas A, Camacho LH, Lopez-Berestein G et al. Antitumor activity in melanoma and anti-self responses in a phase I trial with the anti-cytotoxic T lymphocyte-associated antigen 4 monoclonal antibody CP-675,206. J Clin Oncol 2005; 23: 8968-8977

[46] Carbone DP, Reck M, Paz-Ares L et al. First-Line Nivolumab in Stage IV or Recurrent Non-Small-Cell Lung Cancer. N Engl J Med 2017; 376: 2415-2426

[47] El-Khoueiry AB, Sangro B, Yau T et al. Nivolumab in patients with advanced hepatocellular carcinoma (CheckMate 040): An open-label, non-comparative, phase $1 / 2$ dose escalation and expansion trial. Lancet (London, England) 2017; 389: 2492-2502

[48] Larkin J, Minor D, D'Angelo $S$ et al. Overall survival in patients with advanced melanoma who received nivolumab versus investigator's choice chemotherapy in checkmate 037: A randomized, controlled, open-label phase III trial. J Clin Oncol 2018; 36: 383-390

[49] Maruyama D, Hatake K, Kinoshita T et al. A multicenter phase II study of nivolumab in Japanese patients with relapsed or refractory classical Hodgkin lymphoma. Cancer Sci 2017; 108: 1007-1012

[50] Morris VK, Salem ME, Nimeiri $\mathrm{H}$ et al. Nivolumab for previously treated unresectable metastatic anal cancer (NCI9673): A multicentre, single-arm, phase 2 study. Lancet Oncol 2017; 18: 446-453

[51] Overman M], McDermott R, Leach JL et al. Nivolumab in patients with metastatic DNA mismatch repair-deficient or microsatellite instability-high colorectal cancer (CheckMate 142): An open-label, multicentre, phase 2 study. Lancet Oncol 2017; 18: 1182-1191

[52] Sharma P, Retz M, Siefker-Radtke A et al. Nivolumab in metastatic urothelial carcinoma after platinum therapy (CheckMate 275): A multicentre, single-arm, phase 2 trial. Lancet Oncol 2017; 18: 312-322

[53] Yamazaki N, Kiyohara Y, Uhara H et al. Efficacy and safety of nivolumab in Japanese patients with previously untreated advanced melanoma: A phase II study. Cancer Sci 2017; 108: 1223-1230

[54] Yamazaki N, Kiyohara Y, Uhara H et al. Cytokine biomarkers to predict antitumor responses to nivolumab suggested in a phase II study for advanced melanoma. Cancer Sci 2017; 108: 1022-1031

[55] Antonia S], Lopez-Martin JA, Bendell J et al. Nivolumab alone and nivolumab plus ipilimumab in recurrent small-cell lung cancer (CheckMate 032): A multicentre, open-label, phase 1/2 trial. Lancet Oncol 2016; 17: 883-895

[56] Choueiri TK, Fishman MN, Escudier B et al. Immunomodulatory activity of nivolumab in metastatic renal cell carcinoma. Clin Cancer Res 2016; 22: 5461-5471

[57] Ferris RL, Blumenschein G Jr., Fayette J et al. Nivolumab for recurrent squamous-cell carcinoma of the head and neck. N Engl J Med 2016; 375: $1856-1867$

[58] Gettinger S, Rizvi NA, Chow LQ et al. Nivolumab monotherapy for first-line treatment of advanced non-small-cell lung cancer. J Clin Oncol 2016; 34: 2980-2987

[59] Rizvi NA, Hellmann MD, Brahmer JR et al. Nivolumab in combination with platinum-based doublet chemotherapy for first-line treatment of advanced non-small-cell lung cancer. J Clin Oncol 2016; 34: 2969-2979

[60] Sharma P, Callahan MK, Bono P et al. Nivolumab monotherapy in recurrent metastatic urothelial carcinoma (CheckMate 032): A multicentre, open-label, two-stage, multi-arm, phase $1 / 2$ trial. Lancet Oncol 2016; 17: 1590-1598
[61] Weber J, Gibney G, Kudchadkar R et al. Phase I/II Study of metastatic melanoma patients treated with nivolumab who had progressed after ipilimumab. Cancer Immunol Res 2016; 4: 345-353

[62] Ansell SM, Lesokhin AM, Borrello I et al. PD-1 blockade with nivolumab in relapsed or refractory Hodgkin's lymphoma. N Engl ] Med 2015; 372: 311-319

[63] Borghaei H, Paz-Ares L, Horn L et al. Nivolumab versus docetaxel in advanced nonsquamous non-small-cell lung cancer. N Engl J Med 2015; 373: 1627-1639

[64] Brahmer ], Reckamp KL, Baas P et al. Nivolumab versus docetaxel in advanced squamous-cell non-small-cell lung cancer. $N$ Engl J Med 2015; 373: 123-135

[65] Hamanishi J, Mandai M, Ikeda T et al. Safety and antitumor activity of anti-pd-1 antibody, nivolumab, in patients with platinum-resistant ovarian cancer. J Clin Oncol 2015; 33: 4015-4022

[66] McDermott DF, Drake CG, Sznol M et al. Survival, durable response, and long-term safety in patients with previously treated advanced renal cell carcinoma receiving nivolumab. J Clin Oncol 2015; 33 : 2013-2020

[67] Motzer RJ, Rini BI, McDermott DF et al. Nivolumab for metastatic renal cell carcinoma: results of a randomized phase ii trial. J Clin Oncol 2015; 33: 1430-1437

[68] Rizvi NA, Mazieres J, Planchard D et al. Activity and safety of nivolumab, an anti-PD-1 immune checkpoint inhibitor, for patients with advanced, refractory squamous non-small-cell lung cancer (CheckMate 063): A phase 2, single-arm trial. Lancet Oncol 2015; 16: 257-265

[69] Robert C, Long GV, Brady B et al. Nivolumab in previously untreated melanoma without BRAF mutation. N Engl J Med 2015; 372: 320-330

[70] Weber JS, D'Angelo SP, Minor D et al. Nivolumab versus chemotherapy in patients with advanced melanoma who progressed after anti-CTLA-4 treatment (CheckMate 037): A Randomised, controlled, open-label, phase 3 trial. Lancet Oncol 2015; 16: 375-384

[71] Topalian SL, Sznol M, McDermott DF et al. Survival, durable tumor remission, and long-term safety in patients with advanced melanoma receiving nivolumab. J Clin Oncol 2014; 32: 1020-1030

[72] Bauml J, Seiwert TY, Pfister DG et al. Pembrolizumab for platinumand cetuximab-refractory head and neck cancer: results from a single-arm, phase ii study. J Clin Oncol 2017; 35: 1542-1549

[73] Bellmunt J, de Wit R, Vaughn DJ et al. Pembrolizumab as Second-Line Therapy for Advanced Urothelial Carcinoma. N Engl J Med 2017; 376: 1015-1026

[74] Chen R, Zinzani PL, Fanale MA et al. Phase II study of the efficacy and safety of pembrolizumab for relapsed/refractory classic hodgkin lymphoma. J Clin Oncol 2017; 35: 2125-2132

[75] Delivanis DA, Gustafson MP, Bornschlegl S et al. Pembrolizumab-induced thyroiditis: comprehensive clinical review and insights into underlying involved mechanisms. J Clin Endocrinol Metab 2017; 102: 2770-2780

[76] Ott PA, Piha-Paul SA, Munster P et al. Safety and antitumor activity of the anti-PD-1 antibody pembrolizumab in patients with recurrent carcinoma of the anal canal. Ann Oncol 2017; 28: 1036-1041

[77] Ott PA, Elez E, Hiret $S$ et al. Pembrolizumab in patients with extensive-stage small-cell lung cancer: results from the phase ib keynote-028 study. J Clin Oncol 2017; 35: 3823-3829

[78] Yamazaki N, Takenouchi T, Fujimoto $M$ et al. Phase $1 \mathrm{~b}$ study of pembrolizumab (MK-3475; anti-PD-1 monoclonal antibody) in Japanese patients with advanced melanoma (KEYNOTE-041). Cancer Chemother Pharmacol 2017; 79: 651-660

[79] Zinzani PL, Ribrag V, Moskowitz CH et al. Safety and tolerability of pembrolizumab in patients with relapsed/refractory primary mediastinal large B-cell lymphoma. Blood 2017; 130: 267-270 
[80] Armand P, Shipp MA, Ribrag V et al. Programmed Death-1 Blockade With Pembrolizumab in Patients With Classical Hodgkin Lymphoma After Brentuximab Vedotin Failure. J Clin Oncol 2016; 34: 3733-3739

[81] Chatterjee M, Turner DC, Felip E et al. Systematic evaluation of pembrolizumab dosing in patients with advanced non-small-cell lung cancer. Ann Oncol 2016; 27: 1291-1298

[82] Chow LQ, Haddad R, Gupta S et al. Antitumor activity of pembrolizumab in biomarker-unselected patients with recurrent and/or metastatic head and neck squamous cell carcinoma: results from the phase ib keynote-012 expansion cohort. J Clin Oncol 2016; 34: 3838-3845

[83] de Filette J, Jansen Y, Schreuer M et al. Incidence of thyroid-related adverse events in melanoma patients treated with pembrolizumab. J Clin Endocrinol Metab 2016; 101: 4431-4439

[84] Langer C], Gadgeel SM, Borghaei H et al. Carboplatin and pemetrexed with or without pembrolizumab for advanced, non-squamous non-small-cell lung cancer: A Randomised, phase 2 cohort of the open-label KEYNOTE-021 study. Lancet Oncol 2016; 17: 1497-1508

[85] Muro K, Chung HC, Shankaran V et al. Pembrolizumab for patients with PD-L1-positive advanced gastric cancer (KEYNOTE-012): A multicentre, open-label, phase 1b trial. Lancet Oncol 2016; 17: 717-726

[86] Nanda R, Chow LQ, Dees EC et al. Pembrolizumab in patients with advanced triple-negative breast cancer: Phase Ib KEYNOTE-012 Study. J Clin Oncol 2016; 34: 2460-2467

[87] Reck M, Rodriguez-Abreu D, Robinson AG et al. Pembrolizumab versus Chemotherapy for PD-L1-Positive Non-Small-Cell Lung Cancer. N Engl J Med 2016; 375: 1823-1833

[88] Ribas A, Hamid O, Daud A et al. Association of pembrolizumab with tumor response and survival among patients with advanced melanoma. JAMA 2016; 315: 1600-1609

[89] Seiwert TY, Burtness B, Mehra R et al. Safety and clinical activity of pembrolizumab for treatment of recurrent or metastatic squamous cell carcinoma of the head and neck (KEYNOTE-012): An open-label, multicentre, phase 1b trial. Lancet Oncol 2016; 17: 956-965

[90] Herbst RS, Baas P, Kim DW et al. Pembrolizumab versus docetaxel for previously treated, PD-L1-positive, advanced non-small-cell lung cancer (KEYNOTE-010): A Randomised controlled trial. Lancet (London, England) 2016; 387: 1540-1550

[91] Garon EB, Rizvi NA, Hui R et al. Pembrolizumab for the treatment of non-small-cell lung cancer. N Engl J Med 2015; 372: 2018-2028

[92] Patnaik A, Kang SP, Rasco D et al. Phase I study of pembrolizumab (MK-3475; Anti-PD-1 monoclonal antibody) in patients with advanced solid tumors. Clin Cancer Res 2015; 21: 4286-4293

[93] Ribas A, Puzanov I, Dummer R et al. Pembrolizumab versus investigator-choice chemotherapy for ipilimumab-refractory melanoma (KEYNOTE-002): A Randomised, controlled, phase 2 trial. Lancet Oncol 2015; 16: 908-918

[94] Robert C, Ribas A, Wolchok JD et al. Anti-programmed-death-receptor- 1 treatment with pembrolizumab in ipilimumab-refractory advanced melanoma: A Randomised dose-comparison cohort of a phase 1 trial. Lancet (London, England) 2014; 384: 1109-1117

[95] Balar AV, Galsky MD, Rosenberg JE et al. Atezolizumab as first-line treatment in cisplatin-ineligible patients with locally advanced and metastatic urothelial carcinoma: A Single-arm, multicentre, phase 2 trial. Lancet (London, England) 2017; 389: 67-76

[96] Peters S, Gettinger S, Johnson ML et al. Phase II Trial of Atezolizumab As First-Line or Subsequent Therapy for Patients With Programmed Death-Ligand 1-Selected Advanced Non-Small-Cell Lung Cancer (BIRCH). J Clin Oncol 2017; 35: 2781-2789

[97] Fehrenbacher L, Spira A, Ballinger $M$ et al. Atezolizumab versus docetaxel for patients with previously treated non-small-cell lung cancer (POPLAR): A multicentre, open-label, phase 2 randomised controlled trial. Lancet (London, England) 2016; 387: 1837-1846
[98] McDermott DF, Sosman JA, Sznol M et al. Atezolizumab, an Anti-Programmed Death-Ligand 1 Antibody, in Metastatic Renal Cell Carcinoma: Long-Term Safety, Clinical Activity, and Immune Correlates From a Phase la Study. J Clin Oncol 2016; 34: 833-842

[99] Apolo AB, Infante JR, Balmanoukian A et al. Avelumab, an Anti-Programmed Death-Ligand 1 Antibody, In Patients With Refractory Metastatic Urothelial Carcinoma: Results From a Multicenter, Phase Ib Study. J Clin Oncol 2017; 35: 2117-2124

[100] Gulley JL, Rajan A, Spigel DR et al. Avelumab for patients with previously treated metastatic or recurrent non-small-cell lung cancer (JAVELIN Solid Tumor): Dose-expansion cohort of a multicentre, open-label, phase 1b trial. Lancet Oncol 2017; 18: 599-610

[101] Kaufman HL, Russell J, Hamid O et al. Avelumab in patients with chemotherapy-refractory metastatic Merkel cell carcinoma: A multicentre, single-group, open-label, phase 2 trial. Lancet Oncol 2016; 17: 1374-1385

[102] Powles T, O'Donnell PH, Massard C et al. Efficacy and safety of durvalumab in locally advanced or metastatic urothelial carcinoma: updated results from a phase 1/2 open-label study. JAMA Oncol 2017; 3: e172411

[103] Hellmann MD, Rizvi NA, Goldman JW et al. Nivolumab plus ipilimumab as first-line treatment for advanced non-small-cell lung cancer (CheckMate 012): results of an open-label, phase 1 , multicohort study. Lancet Oncol 2017; 18: 31

[104] Hammers HJ, Plimack ER, Infante JR et al. Safety and efficacy of nivolumab in combination with ipilimumab in metastatic renal cell carcinoma: The CheckMate 016 Study. J Clin Oncol 2017; 35: 38513858

[105] Shoushtari AN, Friedman CF, Navid-Azarbaijani P et al. Measuring toxic effects and time to treatment failure for nivolumab plus ipilimumab in melanoma. JAMA Oncol 2018; 4: 98-101

[106] Wolchok JD, Kluger H, Callahan MK et al. Nivolumab plus ipilimumab in advanced melanoma. $N$ Engl J Med 2013; 369: 122-133

[107] Long GV, Atkinson V, Cebon JS et al. Standard-dose pembrolizumab in combination with reduced-dose ipilimumab for patients with advanced melanoma (KEYNOTE-029): an open-label, phase 1b trial. Lancet Oncol 2017; 18: 1202-1210

[108] Antonia S, Goldberg SB, Balmanoukian A et al. Safety and antitumour activity of durvalumab plus tremelimumab in non-small cell lung cancer: a multicentre, phase 1b study. Lancet Oncol 2016; 17: 299-308

[109] Araujo PB, Coelho MC, Arruda M et al. Ipilimumab-induced hypophysitis: Review of the literature. J Endocrinol Invest 2015; 38: 1159-1166

[110] Corsello SM, Barnabei A, Marchetti P et al. Endocrine side effects induced by immune checkpoint inhibitors. J Clin Endocrinol Metab 2013; 98: 1361-1375

[111] Bertrand A, Kostine M, Barnetche T et al. Immune related adverse events associated with anti-CTLA-4 antibodies: Systematic review and meta-analysis. BMC Medicine 2015; 13: 211

[112] Albarel F, Gaudy C, Castinetti F et al. Long-term follow-up of ipilimumab-induced hypophysitis, a common adverse event of the anti-CTLA-4 antibody in melanoma. Eur J Endocrinol 2015; 172: 195-204

[113] Blansfield JA, Beck KE, Tran K et al. Cytotoxic T-lymphocyte-associated antigen-4 blockage can induce autoimmune hypophysitis in patients with metastatic melanoma and renal cancer. I Immunother (Hagerstown, Md: 1997) 2005; 28: 593-598

[114] Dillard T, Yedinak CG, Alumkal J et al. Anti-CTLA-4 antibody therapy associated autoimmune hypophysitis: Serious immune related adverse events across a spectrum of cancer subtypes. Pituitary 2010; 13: $29-38$ 
[115] Iwama S, De Remigis A, Callahan MK et al. Pituitary expression of CTLA-4 mediates hypophysitis secondary to administration of CTLA-4 blocking antibody. Sci Transl Med 2014; 6: 230ra245

[116] Caturegli P, Di Dalmazi G, Lombardi M et al. Hypophysitis secondary to cytotoxic t-lymphocyte-associated protein 4 blockade: insights into pathogenesis from an autopsy series. Am J Pathol 2016; 186 : 3225-3235

[117] Blomhoff A, Lie BA, Myhre AG et al. Polymorphisms in the cytotoxic $\mathrm{T}$ lymphocyte antigen- 4 gene region confer susceptibility to Addison's disease. J Clin Endocrinol Metab 2004; 89: 3474-3476

[118] Ueda H, Howson JM, Esposito L et al. Association of the T-cell regulatory gene CTLA4 with susceptibility to autoimmune disease. Nature 2003; 423: 506-511

[119] Orlov S, Salari F, Kashat L et al. Induction of painless thyroiditis in patients receiving programmed death 1 receptor immunotherapy for metastatic malignancies. J Clin Endocrinol Metab 2015; 100: 1738-1741

[120] Alhusseini M, Samantray ]. Hypothyroidism in cancer patients on immune checkpoint inhibitors with anti-PD1 Agents: Insights on underlying mechanisms. Exp Clin Endocrinol Diabetes 2017; 125 : 267-269

[121] van Kooten M], van den Berg G, Glaudemans A et al. Transient thyrotoxicosis during nivolumab treatment. Netherl J Med 2017; 75: 204-207

[122] Azmat U, Liebner D, Joehlin-Price A et al. Treatment of ipilimumab induced graves' disease in a patient with metastatic melanoma. Case Rep Endocrinol 2016; 2087525

[123] Min L, Vaidya A, Becker C. Thyroid autoimmunity and ophthalmopathy related to melanoma biological therapy. Eur J Endocrinol 2011 164: 303-307

[124] Gan EH, Mitchell AL, Plummer R et al. Tremelimumab-Induced Graves Hyperthyroidism. Eur Thyr J 2017; 6: 167-170

[125] Andersen TB, Aleksyniene R, Gormsen LC et al. Effect of recent contrast-enhanced CT and patient age on image quality of thyroid scintigraphy. Clin Nucl Med 2015; 40: 297-302

[126] McMillen B, Dhillon MS, Yong-Yow S. A rare case of thyroid storm. BMJ Case Rep 2016, 2016; doi:10.1136/bcr-2016-214603

[127] Morganstein DL, Lai Z, Spain L et al. Thyroid abnormalities following the use of cytotoxic T-lymphocyte antigen- 4 and programmed death receptor protein-1 inhibitors in the treatment of melanoma. Clin Endocrinol 2017; 86: 614-620

[128] Yamauchi I, Sakane Y, Fukuda Y et al. Clinical features of nivolumab-induced thyroiditis: A case series study. Thyroid 2017; 27: 894-901

[129] Angell TE, Lechner MG, Jang JK et al. BRAF V600E in papillary thyroid carcinoma is associated with increased programmed death ligand 1 expression and suppressive immune cell infiltration. Thyroid 2014; 24: $1385-1393$

[130] Chintakuntlawar AV, Rumilla KM, Smith CY et al. Expression of PD-1 and PD-L1 in Anaplastic Thyroid Cancer Patients Treated With Multimodal Therapy: Results From a Retrospective Study. J Clin Endocrinol Metab 2017; 102: 1943-1950

[131] Newby PR, Roberts-Davies EL, Brand O] et al. Tag SNP screening of the PDCD1 gene for association with Graves' disease. Clin Endocrinol 2007; 67: 125-128

[132] Hayashi M, Kouki T, Takasu $\mathrm{N}$ et al. Association of an $\mathrm{A} / \mathrm{C}$ single nucleotide polymorphism in programmed cell death-ligand 1 gene with Graves' disease in Japanese patients. Eur J Endocrinol 2008; 158 : 817-822

[133] Kobayashi T, Iwama S, Yasuda Y et al. Patients with antithyroid antibodies are prone to develop destructive thyroiditis by nivolumab: A prospective study. J Endocr Soc 2018; 2: 241-251
[134] Moeller LC, Fuhrer D. Thyroid hormone, thyroid hormone receptors, and cancer: a clinical perspective. Endocr Relat Cancer 2013; 20: R19-R29

[135] Theodossiou C, Skrepnik N, Robert EG et al. Propylthiouracil-induced hypothyroidism reduces xenograft tumor growth in athymic nude mice. Cancer 1999; 86: 1596-1601

[136] Fabian ID, Rosner M, Fabian I et al. Low thyroid hormone levels improve survival in murine model for ocular melanoma. Oncotarget 2015; 6: 11038-11046

[137] Kinoshita S, Sone S, Yamashita T et al. Effects of experimental hyper- and hypothyroidism on natural defense activities against Lewis lung carcinoma and its spontaneous pulmonary metastases in C57BL/6 mice. Tokushima J Exp Med 1991; 38: 25-35

[138] Baldazzi V, Tassi R, Lapini A et al. The impact of sunitinib-induced hypothyroidism on progression-free survival of metastatic renal cancer patients: A prospective single-center study. Urol Oncol 2012; 30: 704-710

[139] Clemons ], Gao D, Naam M et al. Thyroid dysfunction in patients treated with sunitinib or sorafenib. Clin Genitourin Cancer 2012; 10 225-231

[140] Weijl NI, Van der Harst D, Brand A et al. Hypothyroidism during immunotherapy with interleukin-2 is associated with antithyroid antibodies and response to treatment. J Clin Oncol 1993; 11: 1376-1383

[141] Hercbergs A, Johnson RE, Ashur-Fabian O et al. Medically induced euthyroid hypothyroxinemia may extend survival in compassionate need cancer patients: An Observational study. Oncologist 2015; 20: $72-76$

[142] Matsumura K, Nagasawa K, Oshima Y et al. Aggravation of diabetes, and incompletely deficient insulin secretion in a case with type 1 diabetes-resistant HLA DRB1 * 15:02 treated with nivolumab. J Diabetes Invest 2017; 9: 438-441

[143] Hughes ], Vudattu N, Sznol M et al. Precipitation of autoimmune diabetes with anti-PD-1 immunotherapy. Diabetes Care 2015; 38: e55-e57

[144] Okamoto M, Okamoto M, Gotoh K et al. Fulminant type 1 diabetes mellitus with anti-programmed cell death-1 therapy. J Diabetes Invest 2016; 7: 915-918

[145] Lowe JR, Perry DJ, Salama AK et al. Genetic risk analysis of a patient with fulminant autoimmune type 1 diabetes mellitus secondary to combination ipilimumab and nivolumab immunotherapy. J Immunother Cancer 2016; 4: 89

[146] Fujisawa R, Haseda F, Tsutsumi C et al. Low programmed cell death-1 (PD-1) expression in peripheral CD4(+) T cells in Japanese patients with autoimmune type 1 diabetes. Clin Exp Immunol 2015; 180: 452-457

[147] Perri V, Russo B, Crino A et al. Expression of pd-1 molecule on regulatory $t$ lymphocytes in patients with insulin-dependent diabetes mellitus. Int J Mol Sci 2015; 16: 22584-22605

[148] Luczynski W, Wawrusiewicz-Kurylonek N, Stasiak-Barmuta A et al. Diminished expression of ICOS, GITR and CTLA-4 at the mRNA level in $T$ regulatory cells of children with newly diagnosed type 1 diabetes. Acta Biochim Pol 2009; 56: 361-370

[149] Haseda F, Imagawa A, Murase-Mishiba Y et al. Low CTLA-4 expression in CD4 + helper T-cells in patients with fulminant type 1 diabetes. Immunol Lett 2011; 139: 80-86

[150] Paepegaey AC, Lheure C, Ratour C et al. Polyendocrinopathy resulting from pembrolizumab in a patient with a malignant melanoma. J Endocr Soc 2017; 1: 646-649

[151] Min L, Ibrahim N. Ipilimumab-induced autoimmune adrenalitis. Lancet Diabetes Endocrinol 2013; 1: e15 
[152] Trainer H, Hulse P, Higham CE et al. Hyponatraemia secondary to nivolumab-induced primary adrenal failure. Endocrinol Diabetes Metab Case Reps 2016; 2016: pii 16-0108 [Epub 2016 Nov 1]

[153] Win MA, Thein KZ, Qdaisat A et al. Acute symptomatic hypocalcemia from immune checkpoint therapy-induced hypoparathyroidism. Am J Emerg Med 2017; 35: 1039 e1035-1039 e1037

[154] Horinouchi H, Yamamoto N, Fujiwara Y et al. Phase I study of ipilimumab in phased combination with paclitaxel and carboplatin in Japanese patients with non-small-cell lung cancer. Invest New Drugs 2015; 33: 881-889
[155] Wahab IA, Pratt NL, Kalisch LM et al. The detection of adverse events in randomized clinical trials: Can we really say new medicines are safe? Curr Drug Safety 2013; 8: 104-113

[156] National Cancer Institute. Common Terminology Criteria for Adverse Events (CTCAE).Version 4.03.; June 142010 Available at https:// ctep.cancer.gov/protocoldevelopment/electronic_applications/docs/ CTCAE_4.03.xlsx Accessed March 27, 2018 